\title{
A generic regional spatio-temporal co-occurrence pattern mining model: a case study for air pollution
}

\author{
Mohammad Akbari ${ }^{1,2} \cdot$ Farhad Samadzadegan $^{1}$ • \\ Robert Weibel ${ }^{2}$
}

Received: 9 October 2014/Accepted: 3 June 2015/Published online: 25 June 2015

(C) Springer-Verlag Berlin Heidelberg 2015

\begin{abstract}
Spatio-temporal co-occurrence patterns represent subsets of object types which are located together in both space and time. Existing algorithms for cooccurrence pattern mining cannot handle complex applications such as air pollution in several ways. First, the existing models assume that spatial relationships between objects are explicitly represented in the input data, while the new method allows extracting implicitly contained spatial relationships algorithmically. Second, instead of extracting co-occurrence patterns of only point data, the proposed method deals with different feature types that is with point, line and polygon data. Thus, it becomes relevant for a wider range of real applications. Third, it also allows mining a spatio-temporal co-occurrence pattern simultaneously in space and time so that it illustrates the evolution of patterns over space and time. Furthermore, the proposed algorithm uses a Voronoi tessellation to improve efficiency. To evaluate the proposed method, it was applied on a real case study for air pollution where the objective is to find correspondences of air pollution with other parameters which affect this phenomenon. The results of evaluation confirm not only the capability of this method for co-occurrence pattern mining of complex applications, but also it exhibits an efficient computational performance.
\end{abstract}

Mohammad Akbari

moakbari@ut.ac.ir; mohammad.akbari@geo.uzh.ch

Farhad Samadzadegan

samadz@ut.ac.ir

Robert Weibel

robert.weibel@geo.uzh.ch

1 GIS Division, Department of Surveying and Geomatics Engineering, University College of Engineering, University of Tehran, North Kargar Ave., Tehran, Iran

2 Department of Geography, University of Zurich, Zurich, Switzerland 
Keywords Data mining $\cdot$ Co-occurrence pattern mining $\cdot$ Spatio-temporal $\cdot$ Air pollution

JEL Classification $\mathrm{R} 11 \cdot \mathrm{R} 14 \cdot \mathrm{R} 58 \cdot \mathrm{Q} 53$

\section{Introduction}

Spatial data mining has been introduced to discover interesting and previously unknown, but potentially useful patterns from large spatial databases (Miller and Han 2009; Yoo and Bow 2011). Spatial co-location patterns describe subsets of spatial features which are usually placed in close geographic proximity (Manikandan and Srinivasan 2012b). Furthermore, spatio-temporal co-location pattern mining or co-occurrence pattern mining extends the mining task to the scope of both space and time (Celik et al. 2008; Huang et al. 2008; Qian et al. 2009a, 2009b). Spatial/ spatio-temporal data mining provides a wide range of applications in different fields, such as geographic information systems, geo-marketing, traffic control, database exploration, image processing and environmental studies (Priya et al. 2011). Spatial co-location pattern mining is one of the most important techniques of spatial data mining. It has recently been used to mine the spatial dependencies of objects in different applications (Ding et al. 2008; Xiao et al. 2008). Co-occurrence or spatio-temporal co-occurrence patterns are useful to detect special characteristics behind colocated phenomena (Qian et al. 2009a). To extract interesting and useful patterns from spatio-temporal datasets is more difficult than extracting the corresponding patterns from traditional numerical and categorical data due to the complexity of spatial data types, spatial relationships, spatial autocorrelation and time dependence of events (Wan and Zhou 2008). As a result of these local spatial relationships and the spatial autocorrelation among objects, spatio-temporal cooccurrence patterns have regional properties; therefore, methods that consider this condition as a constraint in their process will yield results reflecting the nature of the given spatial data. Thus, they are more realistic.

Different algorithms have been proposed in both spatial and spatio-temporal cooccurrence pattern mining. In Sect. 2, the review of relevant study and identification of shortcomings will be presented. To overcome these problems of the existing methods, a novel method called RST-CoM is presented to model regional spatiotemporal co-occurrence pattern mining such that:

- A localization step (based on a Voronoi diagram) is used to reduce the search space and the number of candidate patterns which speeds up the mining process;

- A specialized mining process is presented which focuses on so-called pattern core element (PCE) to respond to application domains with emphasis on particular patterns;

- This research extends the algorithm so that it can handle all feature types (points, lines, polygons) in the mining process to generalize the model for all real applications; 
- It mines co-occurrence patterns synchronously in space and time in order to present a fully spatio-temporal model, and it also tracks the evolution of patterns over time by means of a spatio-temporal index to help decision makers with long-term monitoring of patterns;

- The developed method was implemented for a real case study, air pollution pattern mining in Tehran, as a means to help urban management.

The organization of the paper is as follows: The review of related research is presented in Sect. 2. The problem statement is described in Sect. 3, and the proposed algorithm for this research to mine spatio-temporal co-occurrence patterns is described in Sect. 4. The experimental results and their discussion are presented in Sects. 5 and 6, respectively, and the conclusions and perspectives on future study are summarized in Sect. 7.

\section{Literature review}

Recently, the development of many techniques for spatio-temporal co-occurrence pattern mining has gained a significant importance in real-life applications. This research focuses on spatio-temporal data mining, but to achieve the desired model, it is primarily necessary to explore spatial co-location mining models extend them and then propose a new spatio-temporal co-occurrence pattern mining model. Thus, the literature review of this research is organized into two parts. First, it will discuss relevant spatial co-location pattern mining methods followed by a review of spatiotemporal co-occurrence pattern mining techniques.

Various researchers have focused on applying and extending methods for spatial co-location patterns mining in different areas. Several studies focused on global colocation patterns based on a fixed interest measure. Huang et al. (2004) proposed join-based co-location pattern mining, while Yoo and Shekhar (2005, 2006) developed partial-join and join-less mining algorithms using a spatial prevalence criterion as a fixed interest measure. Priya et al. (2011) presented the similarities and differences between the co-location rules problem and the classic association rules and formalized the co-location problem.

Manikandan and Srinivasan (2012a) used an R-tree index for mining co-location patterns to reduce the database search time. Manikandan and Srinivasan study (2012b) proposed a novel algorithm for co-location pattern mining. This method materializes spatial neighborhood relationships with no loss of co-location instances and reduces the computational cost using the aid of Prim's algorithm. In Celik et al. study (2007), a novel and computationally efficient zonal co-location algorithm was presented which used a spatial indexing structure (the clQuad-tree) to store colocations and their instances and to handle dynamic parameters.

In summary, the above approaches have two main shortcomings. First, in some cases, it is necessary to mine patterns with a core element, e.g., co-location patterns of car accident with respect to other affecting parameters. Second, occasionally in real-world applications, parameters of different feature type need to be considered necessitating that in co-location pattern mining, lines and polygons are dealt with in 
addition to points. In fact, there are several studies having partially the same goals. Xiong et al. (2004) proposed a new approach for extended spatial objects (lines and polygons). They used a buffer-based model for mining co-location patterns. However, their method poses some differences: First, they applied co-location pattern mining only for one type of features, i.e., line data, but developed method mines co-location patterns having a combination of point, line and polygon data. Second, their method detects higher-level co-location patterns through costly overlay analysis and a repetitive refinement and combinatorial search. Meanwhile, in this study, using a regional tessellation for pattern core elements and indexing feature instances, it can check all levels of co-location patterns at once. In addition, it uses the participation ratio and participation index to mine interesting patterns which are more efficient compared to their computationally expensive coverage ratio by Xiong et al. (2004). In (Huang et al. 2004), the authors designed an algorithm to discover event centric co-location patterns. They mentioned the event centric model in the paper, but they did not find patterns having focus on a special object. Moreover, they did not mention how an event centric co-location mining model can work with other feature types than points.

Previous literature about mining spatio-temporal co-occurrence patterns can be classified into two categories (Celik et al. 2012): mining of uniform groups of moving objects and mining of mixed groups of moving objects. Using this classification, our problem belongs to the latter one. To mine uniform groups of moving objects, the problems of discovering flock patterns (Gudmundsson and Kreveld 2006) were defined. These methods do not consider feature types and thus are not effective to mine a pattern with different feature types.

With respect to mining mixed groups of moving objects, for discovering partial spatio-temporal co-occurrence patterns, Celik (2011) tried to answer a specific problem. He developed a new method to take the presence period of the objects in the database into account. In addition, for mixed-drove spatio-temporal cooccurrence patterns, Celik et al. (2008) proposed a new monotonic composite interest measure. For cascading spatio-temporal patterns, Mohan et al. (2010) proposed a new method to find partially ordered subsets of event types whose instances are located together and occur in stages. These problems generalize cooccurrence patterns (Huang et al. 2004; Shekhar et al. 2001) (subsets of object types which are frequently located together in space) to the spatio-temporal domain. Furthermore, another approach treated the time factor as an alternative spatial dimension (Huang et al. 2008) to discover the spatio-temporal sequential patterns. However, extremity of all the aforementioned studies is to detect whether a pattern is time prevalent or not, but none of these methods allows to identify how a spatiotemporal co-occurrence pattern evolves over time. Moreover, one key difference of this study is while in the other studies spatial features are tracked as moving objects, in this research, the co-occurrence patterns evolving over time form the moving objects. In other words, the proposed method considers a pattern with all its constitutive elements as a moving object and tracks its evolution instead of each pattern element.

Air pollution can be modeled based on two types of methods, namely land-use regression (LUR) modeling and spatial data mining. There are different researches 
that aimed to model air pollution using LUR. Kanaroglou et al. (2013) developed a spatial autoregressive land-use regression model for sulfur dioxide air pollution concentrations. They have regressed observed $\mathrm{SO}_{2}$ concentrations against a comprehensive set of land-use and transportation variables. Champendal et al. (2014) deal with a new development of nonlinear LUR models based on machine learning algorithms. They assessed the multilayer perceptron and random forest algorithms and their abilities to model the $\mathrm{NO}_{2}$ pollutant in the urban zone of Geneva. Beelen et al. (2013) developed several LUR models in a standardized way in 36 study areas in Europe for the ESCAPE project. They measured nitrogen dioxide $\left(\mathrm{NO}_{2}\right)$ and nitrogen oxides (NOx) in each of the study areas. Moreover, they explained the spatial variation in each area utilizing LUR modeling.

The assessed literatures for LUR modeling reveals that applying these methods needs a comprehensive understanding of each case study application domain. In contrast, the proposed method in the current research was developed based on spatio-temporal concepts that does not need a good knowledge about the case study. Against regression methods that effort to find a function to model the data with the least error, the proposed method attempted to find some rule-like relations between air pollution and affecting parameters. Also, the proposed method extracts and considers air pollution patterns as moving objects during space and time to identify spatio-temporal patterns, while it is a difficult task by LUR modeling.

\section{Problem statement}

\subsection{Preliminaries}

Spatial co-location pattern mining techniques are used to explore subsets of feature types which are frequently located together in space for a given set of feature types, their instances and a neighbor relation $R$ (Celik et al. 2007). To find co-location patterns as described in (Huang et al. 2004), it is necessary to use metrics to measure the desirability of a pattern. A participation ratio $\operatorname{Pr}\left(C, f_{i}\right)$ of feature $f_{i}$ in a colocation $C=\left\{f_{1}, \ldots, f_{j}\right\}, 1 \leq i \leq j$ is the fraction of objects of feature $f_{i}$ in the neighborhood of co-location instances $C-\{f i\}$, where a high participation ratio of an object shows that this feature type is a fundamental element of an extracted colocation. A participation index $P_{i}(C)$ of a co-location $C=\left\{f_{1}, \ldots, f_{j}\right\}, 1 \leq i \leq j$ is defined as $P_{i}(C)=\min _{f_{i} \in C}\left\{\operatorname{Pr}\left(C, f_{i}\right)\right\}$ where a high participation index of a colocation shows that the spatial features of the co-location are more likely to show up together (Yoo and Shekhar 2006).

However, these metrics alone cannot respond to all new emerging applications, all feature types and temporal dimensions inherent to the data. Therefore, new metrics and criteria need to be developed to handle these conditions. To do so, first, this research defines a new problem framework and then provides definitions to model the problem for mining spatio-temporal co-occurrence patterns.

Consider an urban management agency, for instance, they need to control air pollution; therefore, they have to find co-occurrence patterns of air pollution and 
other parameters such as environmental parameters. Hence, it is important to extract patterns in relation to air pollution and also consider all affecting parameters which perhaps are represented by different feature types than points. Therefore, the problem will be to find co-occurrence patterns of studied parameters in relation to a so-called core element parameter (cf. Sect. 3.2).

Figure 1 schematically explains the main elements of a problem framework. As it can be seen in Fig. 1a, in a general spatial problem, there are different data with different feature types. There are point, line and also polygon data. Based on the concept of spatial autocorrelation summarized in Tobler's first law of geography (Goodchild 2003), only spatial co-location pattern mining in local regions seems to be meaningful. Spatially restricting the pattern search not only helps finding meaningful patterns but also significantly reduces the number of co-location mining searches. Therefore, as can be seen in Fig. 1b, a localization method-the Voronoi diagram in our case - can be used to restrict the co-location mining process. Next, however, the method also must have ways of compiling the different feature type instances in each local region in the mining process. That is, it must integrate the parameters based on their closeness to the pattern core element. Thus, as shown in Fig. 1b, a neighborhood radius around the given core elements (depicted by black points) defines circular regions centered around the core elements, with a given radius to represent the neighborhood relation. Subsequently, the pattern mining algorithm needs to intersect the different feature type instances with each neighborhood region of core elements to find interesting co-occurrence patterns.

In addition, it is of paramount importance to mention that finding co-location patterns for a specific time period only may not be highly beneficial. Therefore, this research intends to develop a pattern mining model which considers parameters varying in space and time, thus enabling to find spatio-temporal co-occurrence patterns that change over time as well as tracking their evolution in space and time simultaneously.

\subsection{Basic concepts}

Definition 1 Given a spatial extent, a region is a subset of the spatial extent (Akbari and Samadzadegan 2014).

As (Goodchild 2003) stated "results of analysis vary from one place to another," it is necessary to consider local spatial relationships in mining co-location patterns. The Definition 1 was used to create homogenous non-overlapping regions to apply in co-location mining as presented by Eq. 1:

$$
\mathrm{SD}_{i} \therefore \mathrm{SD}_{i} \subset D,(1 \leq i \leq n) \quad \wedge \quad D=\left\{\mathrm{SD}_{1}, \mathrm{SD}_{2}, \ldots, \mathrm{SD}_{n}\right\}
$$

where $\mathrm{SD}_{\mathrm{i}}$ is a spatial region and $D$ is a spatial extent.

Definition 2 Given a set of spatial features (points, lines or polygons), a pattern core element (PCE) is one of these features, and its instances within the spatial extent are forming the basis of co-location pattern mining. 


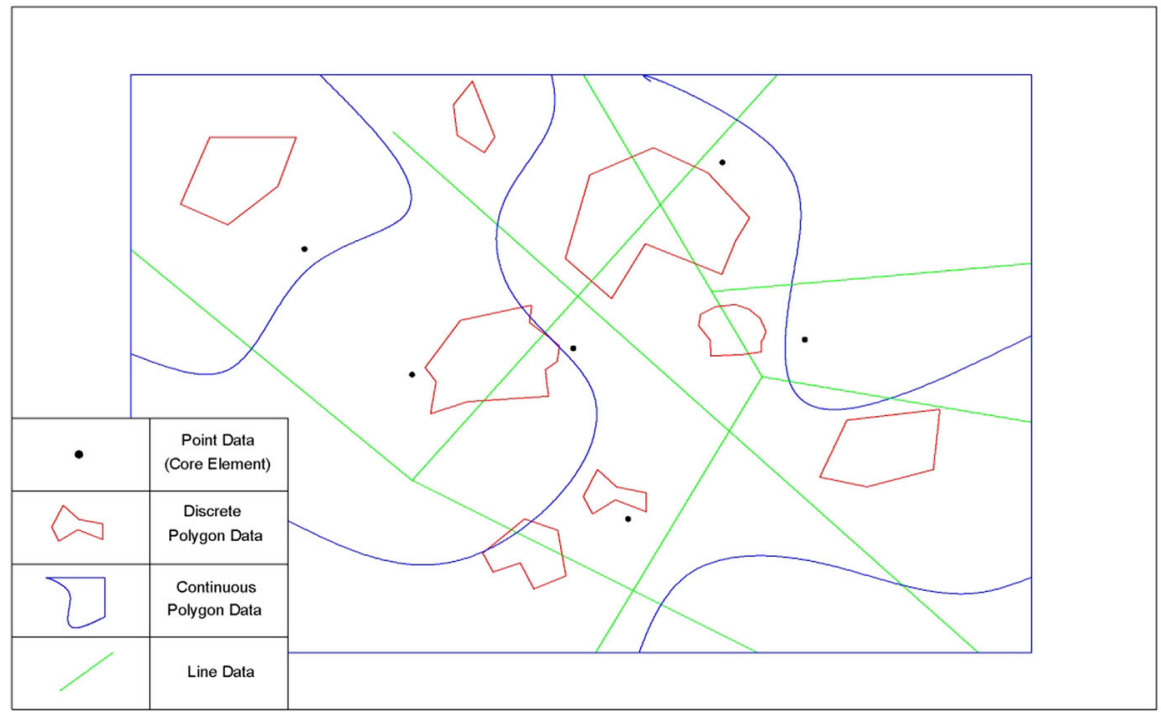

(a)

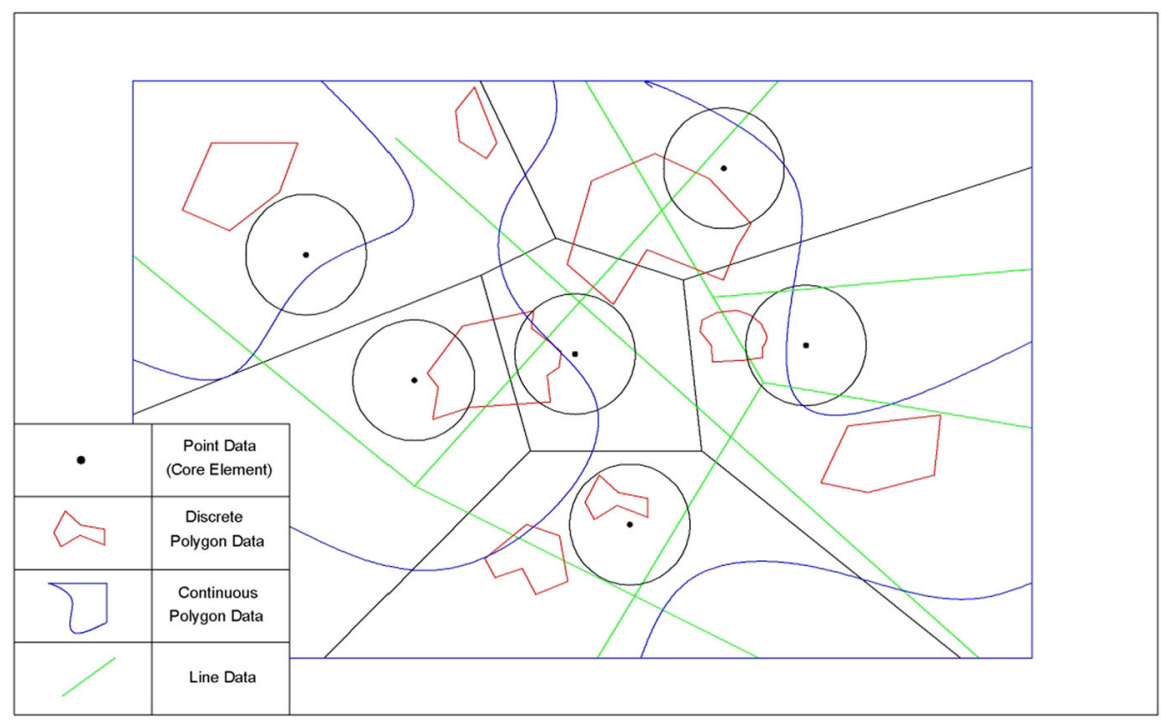

(b)

Fig. 1 a Shows data of different feature types in a geographic space. b Shows the localization using a Voronoi diagram of the core elements, as well as the spatial neighborhood regions of the core elements (depicted by circles)

Consider an application such as air pollution pattern mining. If the task is to find patterns between air pollution and other related parameters such as topography, traffic volume and wind speed, then an air pollution measurement station is a PCE and patterns will be sought regarding it. 
Definition 3 Given a region, a PCE, a set of spatial feature types, their instances and a neighborhood radius $R$, a regional co-location pattern (RCP) is a subset of feature types whose instances are in the neighborhood radius of the PCE in a given region (Akbari and Samadzadegan 2014).

As it is considered, the constrained patterns with PCEs and spatial relationships of objects implicitly (in contrast to existing methods such as Yoo and Shekhar 2006), the proposed method defines a spatial co-location pattern by Definition 3 and Eq. 2.

$$
\mathrm{RCP}=\left\{f_{i} \mid f_{i} \in F \wedge \operatorname{dist}\left(f_{i}, f^{\prime}\right) \leq R\right\}
$$

where, $f_{i}$ : a spatial feature and $1 \leq i \leq m ; f^{\prime}$ : PCE; $\operatorname{dist}\left(f_{i}, f^{\prime}\right)$ : euclidean distance between a spatial feature and a PCE; $R$ : neighborhood radius that is defined based on Euclidean distance around a PCE; $F$ : set of spatial features RCP: regional colocation pattern, that is, a subset of problem spatial features which have close spatial relationships with a PCE.

Definition 4 Given a spatial extent and a set of regions (in our case, Voronoi regions), a PCE is the centroid of each region (Akbari and Samadzadegan 2014).

The method will mine the co-location patterns in the local spaces, which is called regions based on Definition 1 and Definition 3. Due to the challenges of spatial space tessellation mentioned in (Celik et al. 2007), the proposed partitioning and indexing method for this research is the Voronoi diagram. The Voronoi diagram partitions the plane into regions, one for each point site, containing all features of the plane which are closer to this site than to any other (Icking et al. 2003). Therefore, Voronoi diagrams have been formed for PCEs to satisfy Definition 1.

Definition 5 In a co-occurrence pattern mining process, a spatial or temporal prevalent pattern, respectively, is a pattern whose desirability measure (expressed by the participation index or temporal prevalence index, see below) exceeds a given spatial or temporal threshold, respectively.

Mining spatio-temporal co-occurrence patterns needs to consider the temporal dimension as well as spatial one. As mentioned before, considering the time dimension in the mining process needs a metric. Due to lack of a suitable metric, a new temporal metric was proposed that not only can be used in mining prevalent cooccurrence patterns but also can be used to track the evolution trend of patterns. Subsequently, this criterion labeled as temporal prevalence index can be defined as follows.

Definition 6 Given a spatio-temporal dataset, the lifetime dimension is the total number of time slots in the temporal framework of the problem.

Definition 7 Given a spatio-temporal dataset, and a spatial co-location pattern set, the lifetime size of a pattern $C_{k}^{i}, 2 \leq k \leq$ No. of feature - types is the total number of time slots where that pattern is prevalent. 
Definition 8 Given a size-k co-occurrence pattern set PS $=\left\{C_{k}^{1}, \ldots, C_{k}^{n}\right\},(2 \leq k$ $\leq$ No. of feature-types), then, for pattern $C_{k}^{i}$, the temporal prevalence index of $C_{k}^{i}, \operatorname{TPI}\left(\mathrm{PS}, C_{k}^{i}\right)$, is the ratio of the lifetime size of pattern $C_{k}^{i}$ to the lifetime dimension.

\section{RST-CoM: regional spatio-temporal co-occurrence pattern mining model}

The basic idea of regional co-occurrence pattern mining is to index space and reduce search space in order to increase efficiency and achieve results based on spatial and temporal concepts. As Celik et al. (2007) mentioned, common spatial indexing structures are not so applicable for co-location pattern mining. This research applies a spatial index structure formed by the Voronoi tessellation of the PCEs to efficiently mine regional co-location patterns having dynamic factors and also dynamic feature type instances. This eliminates the need to examine objects which are outside the regions of interest (i.e., the Voronoi regions), and the Voronoi index enhances the computational performance. To consider a realistic application context, this model is extended to handle all feature types (points, lines and polygons), thus increasing its generality. In addition, in contrast to the existing models for co-occurrence pattern mining, in this model, a new criterion has been developed that not only can be used to detect spatio-temporal prevalent cooccurrences, but also can be used to identify trends of evolution of patterns in the spatio-temporal framework. Therefore, developing RST-CoM to handle the aforementioned abilities can increase the overall effectiveness of the co-location mining process with respect to time dimension. Algorithm 1 gives the pseudo-code of the RST-CoM algorithm. In the RST-CoM algorithm (cf. pseudo-code in Algorithm 1), line 1 initializes the parameters. Line 2 invokes the localization of the spatial extent. Line 3 through 13 represents the core of the algorithm and is explained in detail below; and line 14 returns the results.

Before describing the actual algorithm, the localization step in line 2 warrants more detail. The PCEs may be of different feature types (point, line and polygon). If the PCEs are point data, then loc_space () creates the Voronoi regions based on the point PCEs and spatially indexes the features to the Voronoi polygons to localize each feature to a limited search space. Therefore, when it intends to check different objects in the neighborhood of a PCE, only the features indexed to that local Voronoi polygon are considered. Alternatively, if the PCEs are line or polygon data, then loc_space () works in a different way. To handle these types of PCEs, we introduce the concept of an MNBR (minimum neighborhood bounding rectangle). It is defined based on the case in point PCEs. In this case, loc_space () works as follows: First, using a Euclidean distance $R$ around the feature instances creates a neighborhood space. Second, a minimum bounding rectangle is generated around the neighborhood space of the PCE: the MNBR. Third, these MNBRs are used as local regions to index features. Figure 2 depicts the neighborhood space (dashed line) and the MNBR (solid line) around a polyline and a polygon PCE, respectively. 


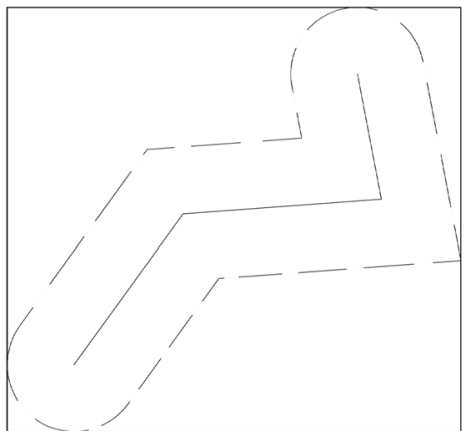

(a)

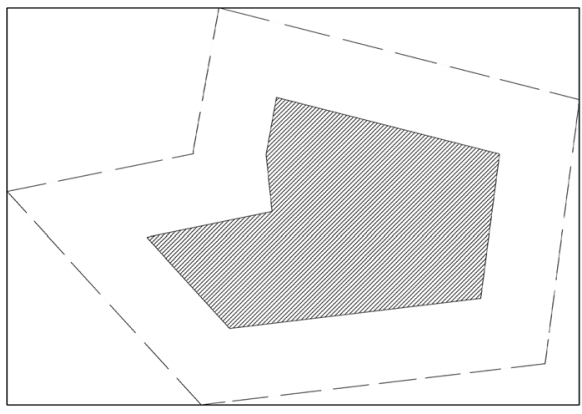

(b)

Fig. 2 a MNBR for polyline PCE. b MNBR for polygon PCE

It is noteworthy that for the purposes of this paper, only the case of point PCEs will be considered.

\section{Algorithm 1: RST-CoM \\ Inputs}

F: a set of distinct spatial feature types,

FI: a set of feature type instances

PCE: a set of pattern core elements

ST: a spatio-temporal dataset

TF: a time slot framework $\left\{t_{0}, \ldots, t_{n-1}\right\}$

$\boldsymbol{\theta}_{\boldsymbol{s}}: \quad$ a spatial prevalence threshold

$\boldsymbol{\theta}_{t}: \quad$ a temporal prevalence threshold

\section{Output:}

- Spatio-temporal co-occurrence patterns whose spatial and temporal prevalence indices are greater than $\theta_{s}$ and $\theta_{t}$, respectively.

- Spatio-temporal evolution of co-occurrence patterns in temporal framework (TF)

\section{Variables:}

k: $\quad$ co-occurrence size

t: $\quad$ time slots $(0, \ldots, \mathrm{n}-1)$

R: $\quad$ spatial neighborhood relationship

$\mathbf{C}_{\mathbf{k}}$ : $\quad$ set of candidate size-k co-locations

$\mathbf{I}_{\mathbf{k}}$ : $\quad$ set of instances of size-k co-locations

$\mathbf{S C P}_{\mathbf{k}}$ : set of spatially prevalent size-k co-locations

$\mathbf{T P I}_{\mathbf{k}}$ : set of temporal prevalence indices of size-k co-locations

$\mathbf{S T C}_{\mathbf{k}}$ : set of spatio-temporal size-k co-occurrences

$\mathbf{P M}_{\mathbf{k}}$ : set of spatio-temporal evolution of size-k co-occurrences 
Inputs:

F: a set of distinct spatial feature types,

FI: a set of feature type instances

PCE: a set of pattern core elements

ST: a Spatio-Temporal dataset

TF: a time slot framework $\left\{t_{0}, \ldots, t_{n-1}\right\}$

$\boldsymbol{\theta}_{s}$ : a spatial prevalence threshold

$\boldsymbol{\theta}_{\boldsymbol{t}}$ : a temporal prevalence threshold

\section{Output:}

- Spatio-Temporal Co-occurrence patterns whose spatial and temporal prevalence indices are greater than $\theta_{s}$ and $\theta_{t}$, respectively.

- Spatio-Temporal evolution of Co-occurrence patterns in Temporal framework (TF)

\section{Variables:}

k: co-occurrence size

$\mathrm{t}$ : time slots $(0, \ldots, \mathrm{n}-1)$

R: spatial neighborhood relationship

$C_{k}$ : set of candidate size $\mathrm{k}$ co-locations

$\mathrm{l}_{\mathrm{k}}$ : set of instances of size $\mathrm{k}$ co-locations

$\mathrm{SCP}_{\mathrm{k}}$ : set of spatially prevalent size $\mathrm{k}$ co-locations

$\mathrm{TPl}_{\mathrm{k}}$ : set of temporal prevalence indices of size $\mathrm{k}$ co-locations

$S T C_{k}$ set of Spatio-Temporal size k co-occurrences

$\mathrm{PM}_{\mathrm{k}}$ : set of Spatio-Temporal evolution of size $\mathrm{k}$ co-occurrences 


\section{Algorithm}

1: Initialization, $k=1, C_{k}=F, S T C_{k}=S T$

2: loc_space (PCE, F, FI)

3: while (not empty $S T C_{k}$ )

4: $\quad C_{k+1}=$ gen_co-occurrence_candidates $\left(C_{k}, S T C_{k}\right)$

5: for each time slot $t(0<t<n-1)$

6: $\quad l_{k+1}(t)=$ gen_co-occurrence_instances $\left(C_{k+1}(t), I_{k}(t), R\right)$

7: $\quad S C P_{k+1}(t)=$ mine_spatial_prev_co-occurrences $\left(C_{k+1}(t), I_{k+1}(t), \theta_{S}\right)$

8: $\quad$ end for

9: $\quad T P I_{k+1}=$ calc_temporal_prev_indices $\left(S C P_{k+1}, T F\right)$

10: $\quad S T C_{k+1}=$ mine_ST_prev_co-occurrences $\left(S C P_{k+1}, T P_{k+1}, \theta_{t}\right)$

11: $\quad \mathrm{PM}_{\mathrm{k}+1}=$ mine_ST_evolution_co-occurrences $\left(\mathrm{TPI}_{\mathrm{k}+1}\right)$

$12: k=k+1$

13: end while

14: return $\left\{S T C_{2}, \ldots, S T C_{k+1}, \mathrm{PM}_{2}, \ldots, \mathrm{PM}_{\mathrm{k}+1}\right\}$

This algorithm has several functions that can be explained as follows. In line 4, the function gen_co-occurrence_candidates() generates size- $k+1$ candidate cooccurrence patterns $C_{k+1}$ for each time slot based on all size- $k$ pattern using an apriori-based method (Agarwal and Srikant 1994). In line 6, the function gen_cooccurrence_instances() works similarly to (Huang et al. 2004) by joining neighbor instances of size- $k$ spatio-temporal co-occurrence patterns for each time slot, generating the instances of candidate $C_{k+1}$. In line 7, the function mine_spatial_prev_co-occurrences () evaluates the candidates to find those patterns whose spatial prevalence criteria are greater than a threshold $\left(\theta_{s}\right)$. The spatial prevalence criterion of patterns in this research is the participation index such as in (Huang et al. 2004), but as mentioned, since it should handle all feature types in the co- 
location mining process, it is necessary to extend the existing criteria. Therefore, new participation ratios were developed according to the following Eqs. 3, 4 and 5. The participation index then is the minimum of the participation ratios of all feature types in a co-location.

$$
\text { For point data: } \operatorname{Pr}\left(C, f_{i}\right)=\frac{\mathrm{NI}\left(f_{i}\right)}{N\left(f_{i}\right)}
$$

where $\mathrm{NI}\left(f_{i}\right)$ is the number of $f_{i}$ feature instances in co-location instance neighborhoods of $C$ and $N\left(f_{i}\right)$ is the total number of $f_{i}$ feature instances.

$$
\text { For line data: } \operatorname{Pr}\left(C, f_{i}\right)=\frac{\mathrm{NI}\left(f_{i}\right)}{N\left(f_{i}\right)}+\frac{\left(l_{1} w_{1}+l_{2} w_{2}+\ldots\right)}{N\left(f_{i}\right) \times\left(w_{1}+w_{2}+\ldots\right)}
$$

where $\mathrm{NI}\left(f_{i}\right)$ is the number of $f_{i}$ feature instances that are completely contained in co-location instance neighborhoods of $C$ and $N\left(f_{i}\right)$ is the total number of $f_{i}$ feature instances. In addition, $l_{i}$ is the number of line features of which $w_{i}$ fraction of length $\left(0<w_{i}<1\right)$ falls inside of co-location instance neighborhoods of $C$.

$$
\text { For polygon data: } \operatorname{Pr}\left(C, f_{i}\right)=\frac{\mathrm{NI}\left(f_{i}\right)}{N\left(f_{i}\right)}+\frac{\left(l_{1} s_{1}+l_{2} s_{2}+\ldots\right)}{N\left(f_{i}\right) \times\left(s_{1}+s_{2}+\ldots\right)}
$$

where $\mathrm{NI}\left(f_{i}\right)$ is the number of $f_{i}$ feature instances that are completely contained in co-location instance neighborhoods of $C$ and $N\left(f_{i}\right)$ is the total number of $f_{i}$ feature instances. In addition, $l_{i}$ is the number of polygon features of which $s_{i}$ fraction of area $\left(0<s_{i}<1\right)$ falls inside of co-location instance neighborhoods of $C$.

Using a for loop, the algorithm finds size- $k+1$ spatially prevalent co-occurrence patterns for each time slot. In line 9, the function calc_temporal_prev_indices(), based on definitions 6,7 and 8, calculates size- $k+1$ temporal prevalence indices of all spatially prevalent patterns size- $k+1$. Then, in line 10 , the function mine_ST_prev_co-occurrences() finds all size- $k+1$ spatially prevalent co-occurrences whose temporal prevalence index meets the temporal threshold $\left(\theta_{t}\right)$. Finally, in line 11, the function mine_ST_evolution_co-occurrences() assesses the status of different size- $k+1$ spatio-temporal co-occurrence patterns and classifies them into six states. This categorization takes place based on the patterns' temporal prevalence indices (TPI), simultaneously considering spatial prevalence, since as stated in Definitions 7 and 8, it is introduced based on the spatial participation index (PI):

- If for the temporal framework $\mathrm{TF}:\left[t_{0}, t_{n-1}\right] \rightarrow \operatorname{TPI}\left(C_{k}\right)=1$, then the pattern is SUSTAINED

- If $\exists t_{m}, m \in(1,2, \ldots, n-1)$ so that for $\left[0, t_{m}\right) \rightarrow \operatorname{TPI}\left(C_{k}\right)=0$, and for $\left[t_{m}, t_{n-1}\right] \rightarrow \operatorname{TPI}\left(C_{k}\right)>0$, then the pattern is EMERGING

- If $\exists t_{m}, m \in(1,2, \ldots, n-1)$ so that for $\left[0, t_{m}\right) \rightarrow \operatorname{TPI}\left(C_{k}\right)>0$ and for $\left[t_{m}, t_{n-1}\right] \rightarrow \operatorname{TPI}\left(C_{k}\right)=0$, then the pattern is DISPERSING

- If for the temporal framework $\mathrm{TF}:\left[t_{0}, t_{n-1}\right] \rightarrow 0.5 \leq \mathrm{TPI}\left(C_{k}\right)<1$, then the pattern is TIME PREVALENT 
- If for the temporal framework $\mathrm{TF}:\left[t_{0}, t_{n-1}\right] \rightarrow 0<\operatorname{TPI}\left(C_{k}\right)<0.5$, then the pattern is TIME VARIANT

- If for the temporal framework TF : $\left[t_{0}, t_{n-1}\right] \rightarrow \operatorname{TPI}\left(C_{k}\right)=0$, then there is $\underline{\mathrm{NO}}$ PATTERN.

\section{Experimental evaluation}

In this section, the proposed RST-CoM algorithm was evaluated experimentally with real-world data, with the aim of spatio-temporal co-occurrence pattern mining of air pollution against external drivers and influences, including road traffic, wind and topography.

\subsection{Datasets}

The datasets used include air pollution, meteorology, traffic and topography of part of Tehran (for more information, see Table 1). The study area can be seen in Fig. 3. The study area consists of regions number 1-8, 21 and 22 of Tehran city (depicted in different colors in Fig. 3). These regions were selected because data were primarily available for these units, and secondly, the data were up-to-date and had enough spatial and temporal overlap. The data span 12 days of different months between March 21, 2011, and March 19, 2012 (a Solar Hijri Year), 1 day per month.

A selection of feature types in the air pollution pattern mining problem was used as shown in Table 2. The raw data were preprocessed to yield the input data for the pattern mining process. Datasets were prepared for each feature type that included the classification of feature type, time information and spatial position information. As can be seen in Table 2, each feature type was classified into three different classes to apply them directly to the mining process (refer to Table 2 for details about the classification). Class breaks were selected after studying the distribution characteristics of each parameter. For traffic data, the classification is based on the

Table 1 Information about applied data in this research

\begin{tabular}{|c|c|c|c|c|c|}
\hline Parameter & $\begin{array}{l}\text { Feature } \\
\text { type }\end{array}$ & $\begin{array}{l}\text { No. of } \\
\text { objects }\end{array}$ & $\begin{array}{l}\text { No. } \\
\text { measurement } \\
\text { stations }\end{array}$ & $\begin{array}{l}\text { Temporal } \\
\text { resolution }\end{array}$ & Source \\
\hline $\begin{array}{l}\text { Topography } \\
\text { (DEM) }\end{array}$ & Polygon & 264 & - & - & $\begin{array}{l}\text { National Cartographic } \\
\text { Center of Iran }\end{array}$ \\
\hline $\begin{array}{l}\text { Air pollution (CO- } \\
\text { pollutant) }\end{array}$ & Point & 108 & 9 & Daily & $\begin{array}{l}\text { Tehran Air Quality } \\
\text { Control Center }\end{array}$ \\
\hline $\begin{array}{l}\text { Meteorology } \\
\text { (wind Speed) }\end{array}$ & Polygon & 36 & 3 & Daily & $\begin{array}{l}\text { Iran Meteorological } \\
\text { Organization }\end{array}$ \\
\hline Road traffic & Line & 20136 & 700 & Daily & $\begin{array}{l}\text { Tehran Traffic Control } \\
\text { Center }\end{array}$ \\
\hline
\end{tabular}




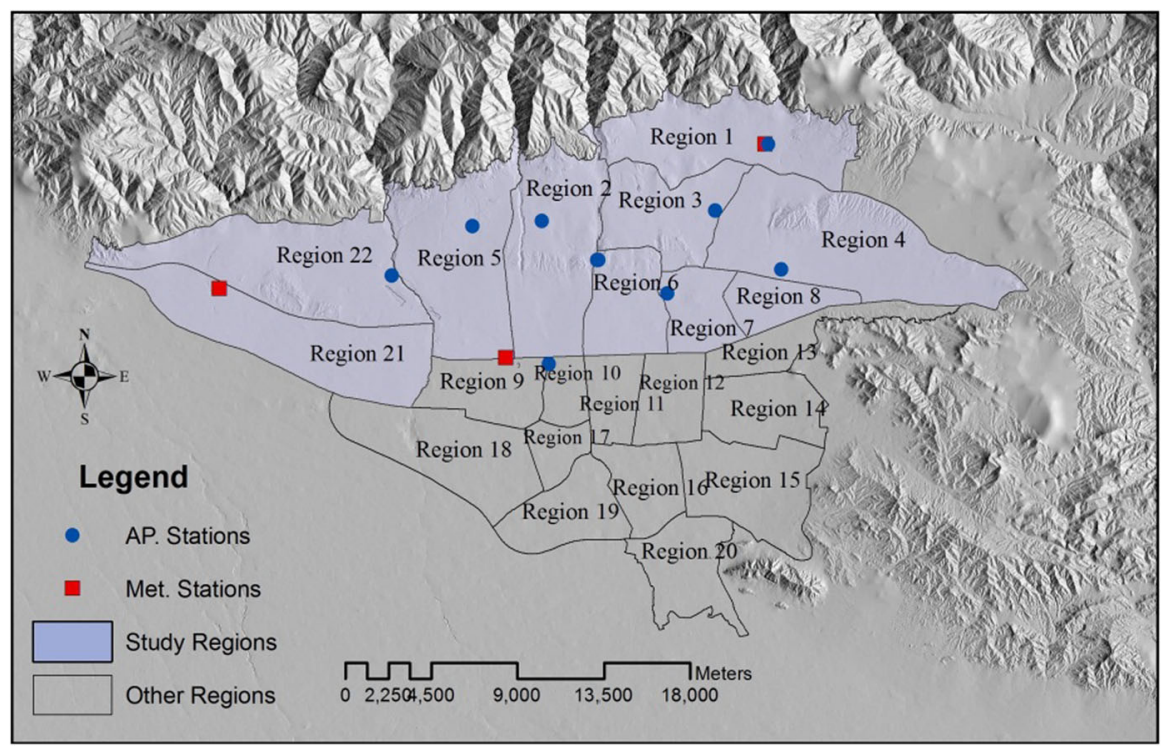

Fig. 3 Study area

Table 2 Detailed classification of case study parameters

\begin{tabular}{|c|c|c|c|c|c|c|c|}
\hline Class & Subclass & Quantity domain & Label & Class & Subclass & $\begin{array}{l}\text { Quantity } \\
\text { domain }\end{array}$ & Labe \\
\hline \multirow{3}{*}{$\begin{array}{l}\text { Traffic } \\
\text { volume }\end{array}$} & Fluent & Fluent traffic & $\operatorname{Tr} 1$ & Topography & Down & $<1300$ & Tp1 \\
\hline & $\begin{array}{l}\text { Semi- } \\
\text { heavy }\end{array}$ & $\begin{array}{l}\text { Disruption in } \\
\text { movement/heavy but } \\
\text { moving traffic }\end{array}$ & $\operatorname{Tr} 2$ & & Medium & $1300-1500$ & $\mathrm{Tp} 2$ \\
\hline & Heavy & Heavy/very heavy traffic & $\operatorname{Tr} 3$ & & High & $>1500$ & Tp3 \\
\hline \multirow{3}{*}{$\begin{array}{l}\text { Wind } \\
\text { speed }\end{array}$} & Low & $<6$ & Wn1 & Co-pollutant & Low & $<1$ & Ap1 \\
\hline & Normal & $6-16$ & Wn2 & & Normal & $1-5$ & Ap2 \\
\hline & High & $>16$ & Wn3 & & High & $>5$ & Ap3 \\
\hline
\end{tabular}

original five classes used by the Tehran Traffic Control Center: fluent, disruption in movement, heavy but moving, heavy and very heavy. The original five classes were summarized into three classes: fluent, semi-heavy and heavy. Similarly for air pollution, since data were used from the Tehran Air Quality Control Center, their rules for the classification of pollutants were adopted. This center, on its Web site, states the classification for each air pollutant. In this research, $\mathrm{CO}$ data were applied and classified accordingly. For the meteorological parameters, wind data were used, since it is the meteorological factor that affects air pollution the most (Table 2). The raw data were obtained from the Iran Meteorological Organization. To classify the wind data based on this organization's proposal, the Beaufort scale was used. Finally, for the classification of topography, because Tehran lies on the hill slope of 
the Alborz Mountains and its height values differ almost uniformly linear along a cross section, three meaningful classes were formed after studying the height variation over Tehran. The detailed class boundaries are shown in Table 2 along with the class labels that were used in the mining process.

\subsection{Implementation results}

As aforementioned, to evaluate the proposed algorithm and also to extract useful patterns of air pollution with the other affecting parameters in Tehran City, the algorithm was implemented in C\#. The implemented interface has four tabs: (1) data view to show and see data and also results; (2) RST-CoM panel, which enables to enter the desired input values such as neighborhood radius, spatial prevalence threshold, temporal prevalence threshold and temporal duration; (3) pattern visualization panel to visualize the extracted co-occurrence patterns in each time slot, which allows to select a desired time slot and visualize the corresponding spatio-temporal co-occurrence patterns in the spatial domain (i.e., on a map); (4) region statistics panel, which serves to extract some information for different regions and time slots.

Due to gaps in the time series data, the fourth day of each month over 1 year was selected, thus yielding totally 12 days. In fact, Persian months were considered and different months were checked for their work days (in Persian weeks, workdays are from Saturday until Wednesday). The fourth day of the month was then selected because it falls mostly on a workday throughout the year and also avoids data gaps. The following input values were used.

- Neighborhood radius: $2000 \mathrm{~m}$; this radius was selected based on the average distance between air pollution measurement stations and spatially meaningful variation of affecting parameters in a neighborhood.

- Spatial prevalence threshold: 0.5; this value was selected based on the idea that a pattern is prevalent if its importance criterion supplies at least for half the participant features.

- Time prevalence threshold: 0.6; this is selected somewhat higher than $50 \%$ such that only the most important patterns are found.

The pattern mining with RST-CoM applied to the case study leads to interesting results (Fig. 4). In Fig. 4, some information for each mined time slot has been summarized. For example, in Fig. 4, there is a pattern (ap2; tr1) that is depicted with dark pink color. This pattern can be found in all time slots. This means that there is a pattern between air pollution type 2 (ap2 in Table 2 ) and road traffic type 1 ( $\operatorname{tr} 1$ in Table 2) in all studied time slots. The explanation for the other patterns in Fig. 4 works accordingly. In other words, Fig. 4 shows extracted patterns in different time slots in a column chart with different colors, where patterns which have common parts have similar colors.

In addition, the spatial visualization of extracted patterns for each time slot is shown in Fig. 5. In the spatial representation of the extracted patterns, a similar method as introduced by Desmier et al. (2011) for the visualization of co-location 


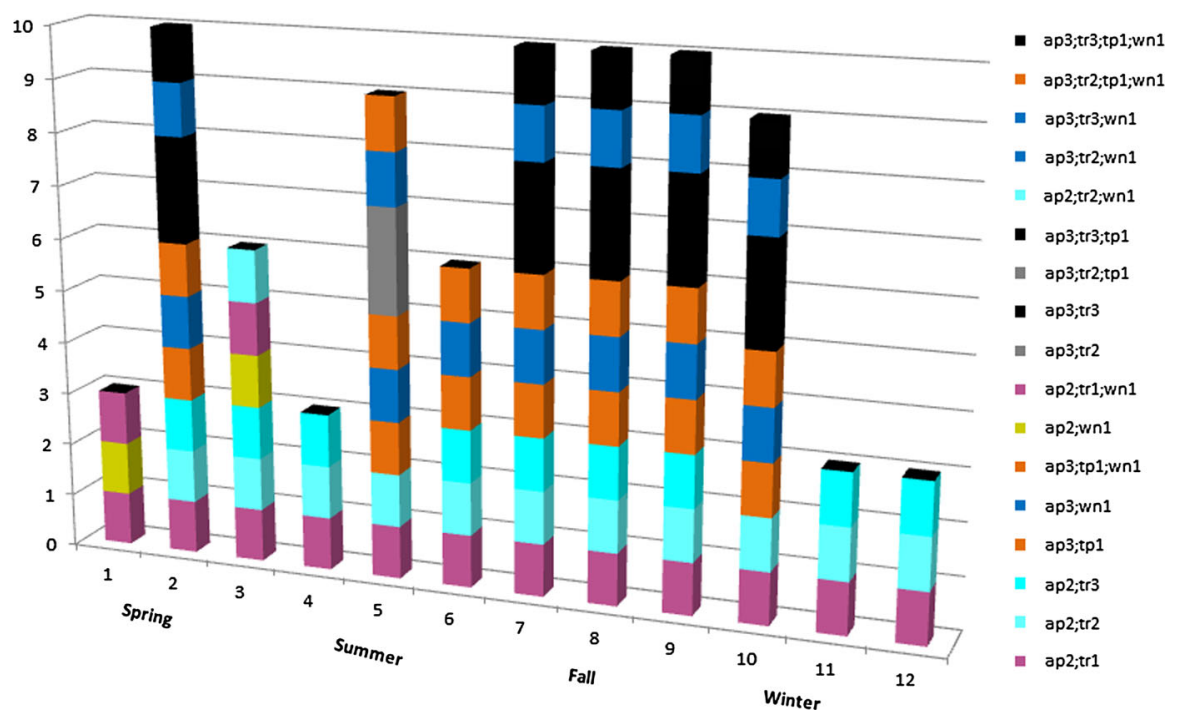

Fig. 4 Pattern distribution in different time slots

patterns was applied. However, this research has made some extensions to the original method of Desmier et al. (2011) to handle data of all feature types. In Fig. 5, each illustrated pattern is a surrogate of all similar prevalent occurring patterns in the spatial extent of the case study. To distinguish each pattern from the others, different colors were used, and to show the importance of a pattern, the line width was varied according to the associated PI value.

Of the 60 different assessed candidate patterns that have had 473 compositions in 12 time slots, there were

- 1 Sustained pattern $\{(\operatorname{ap} 2 ; \operatorname{tr} 1)\}$

- 5 Time prevalent patterns $\{(\operatorname{ap} 2 ; \operatorname{tr} 3),(\operatorname{ap} 2 ; \operatorname{tr} 2),(\operatorname{ap} 3 ; \operatorname{tp} 1),(\operatorname{ap} 3 ;$ wn1 $),(\operatorname{ap} 3$; tp1; wn1)\}

- 11 Time variant patterns \{(ap2;wn1), (ap2;tr1;wn1), (ap3;tr2), (ap3;tr3), (ap3;tr2;tp1), (ap3;tr3;tp1), (ap2;tr2;wn1), (ap3;tr2;wn1), (ap3;tr3;wn1), (ap3;tr2;tp1;wn1), (ap3;tr3;tp1;wn1)\}

- And finally, 43 candidates that showed no particular pattern (i.e., exceeded none of the thresholds).

To assess the performance of the proposed model, an efficiency test was conducted on a notebook with the following software and hardware configuration:

- Windows 7 Ultimate

- Dual core processor

- $1.86 \mathrm{GHz} \mathrm{CPU}$

- 2 GB DDR2 SDRAM 


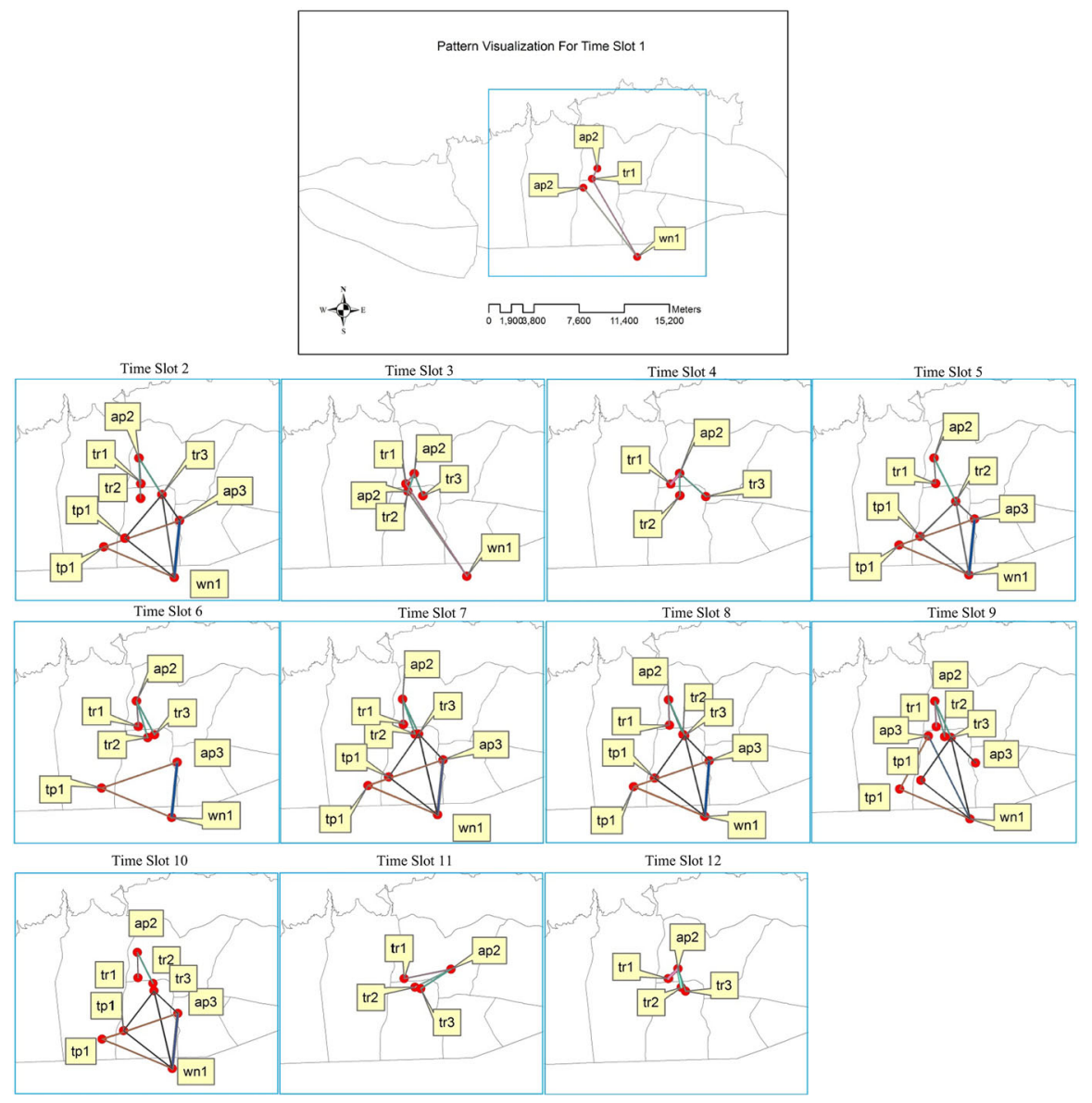

Fig. 5 Spatial visualization of extracted patterns for different time slots

- $160 \mathrm{~GB}(5400 \mathrm{rpm}) \mathrm{HDD}$

- $1 \mathrm{MB}$ Cache.

The proposed model was evaluated with different neighborhood radiuses. As can be seen in Fig. 6, five different neighborhood radii, 500, 1000, 1500, 2000 and $2500 \mathrm{~m}$ were applied. All other parameters were left unchanged (number of time slots $=6$, spatial prevalence threshold $=0.5$, temporal prevalence threshold $=0.6$ ). Figure 6 then plots the processor time against different neighborhood radiuses. Two different regression models were then fitted, a linear model (shown in Fig. 6a) and a quadratic model (Fig. 6b). In fact, $R^{2}$ value in Fig. $6 \mathrm{~b}$ shows a better fitting compared to Fig. 6a, especially when the radius increases much more than $2500 \mathrm{~m}$, the fitting will be quadratic. However, in this studied interval, it can be 
considered as a linear fitting and this research can claim that the proposed model is of the order of $\mathrm{O}(\mathrm{n})$. It is also necessary to mention that radiuses bigger than $3000 \mathrm{~m}$ for this case study were not meaningful since the neighborhood regions start overlapping (the distance between the air pollution measurement stations is never larger than $5800 \mathrm{~m}$ ). Furthermore, air pollution neighborhood search beyond this range is not effective since the maximum variance of pollution has already been reached at this range.

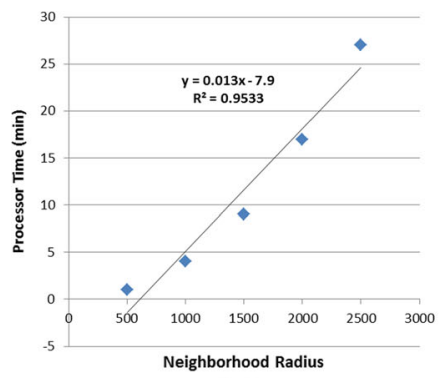

(a)

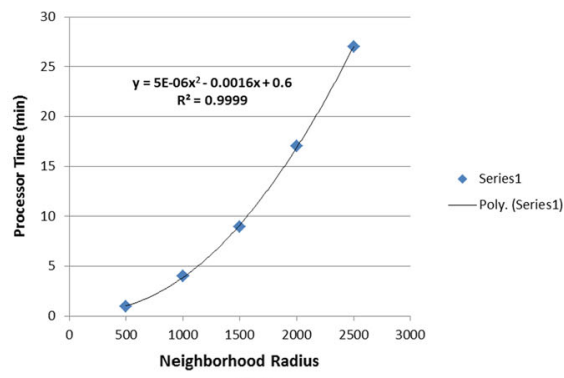

(b)

Fig. 6 Variation of processor time against different neighborhood radii a linear equation fitting b polynomial equation fitting

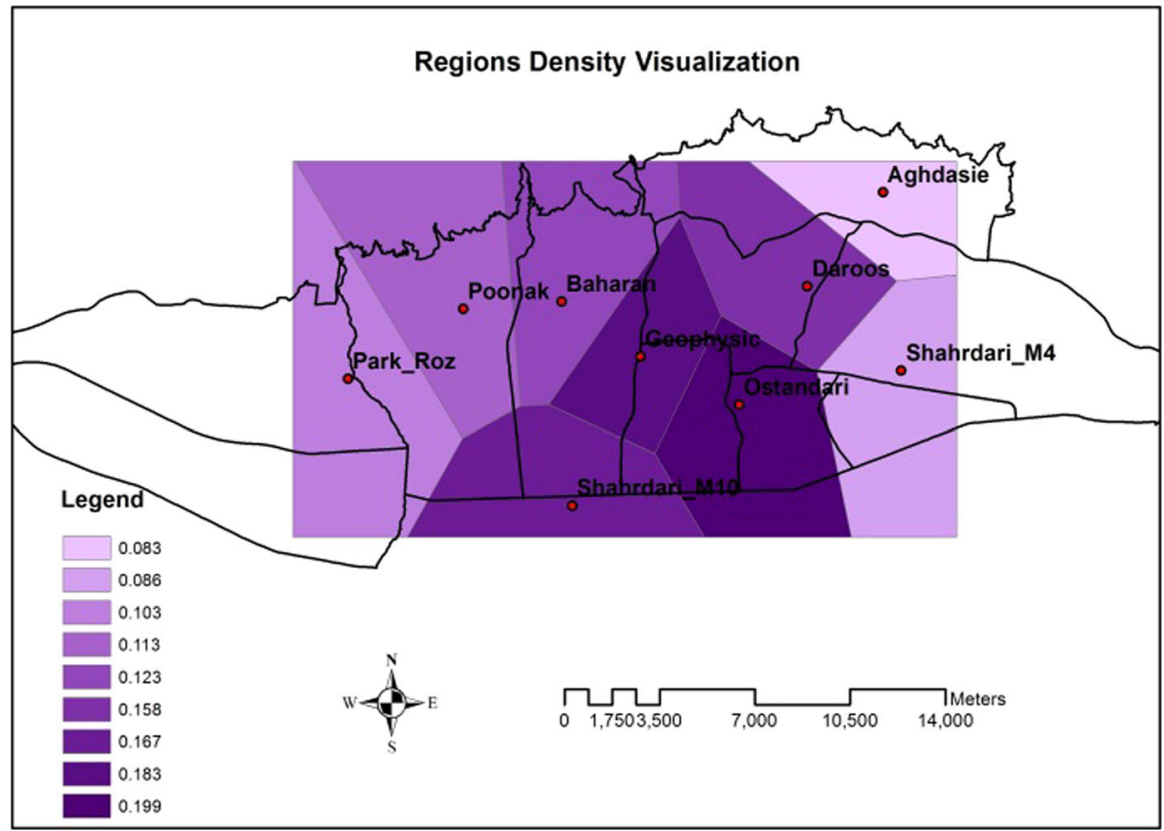

Fig. 7 Map of the VDF index for the case study 
As mentioned above, in regions statistics tab of the developed model, three criteria were presented including VDF (Voronoi density function), VGF (Voronoi gravity function) and VIF (Voronoi impact function). These criteria can be calculated based on the results of RST-CoM algorithm. They can be defined as follows:

$$
\begin{gathered}
\mathrm{VDF}_{i}=\frac{\text { Number of spatial feature instances in } i \text { th Voronoi region }}{\text { Total Number of spatial feature instances in spatial framework }} \\
\mathrm{VGF}_{i}=\frac{\text { Number of co-location instances in } i \text { th Voronoi region }}{\text { Total Number of co-location instances in spatial framework }} \\
\mathrm{VIF}_{i}=\frac{\sum_{j=1}^{m}(\text { Weighted co-location patterns in } i \text { th Voronoi region })}{m}
\end{gathered}
$$

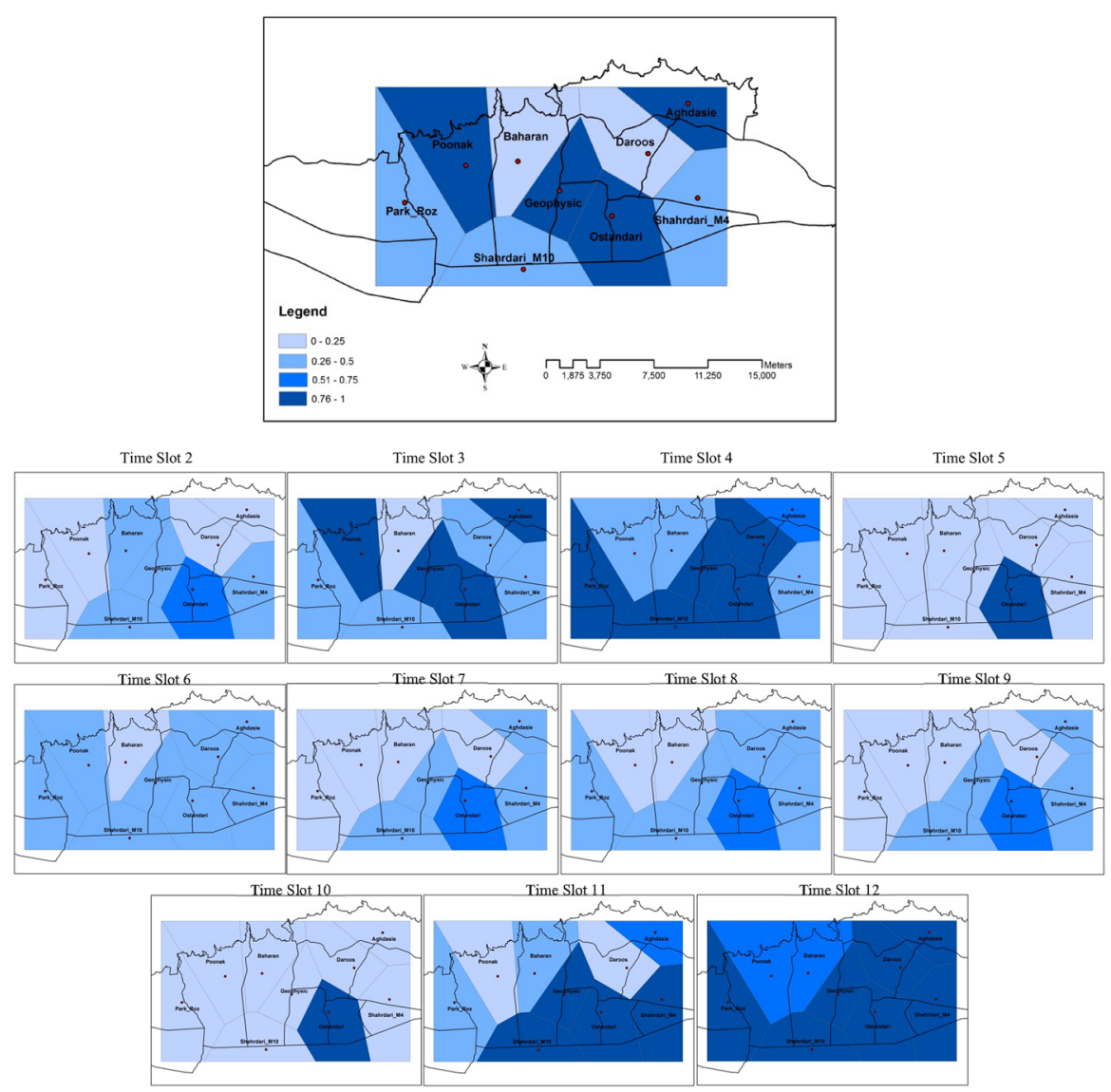

Fig. 8 Map of the VGF index for different time slots 


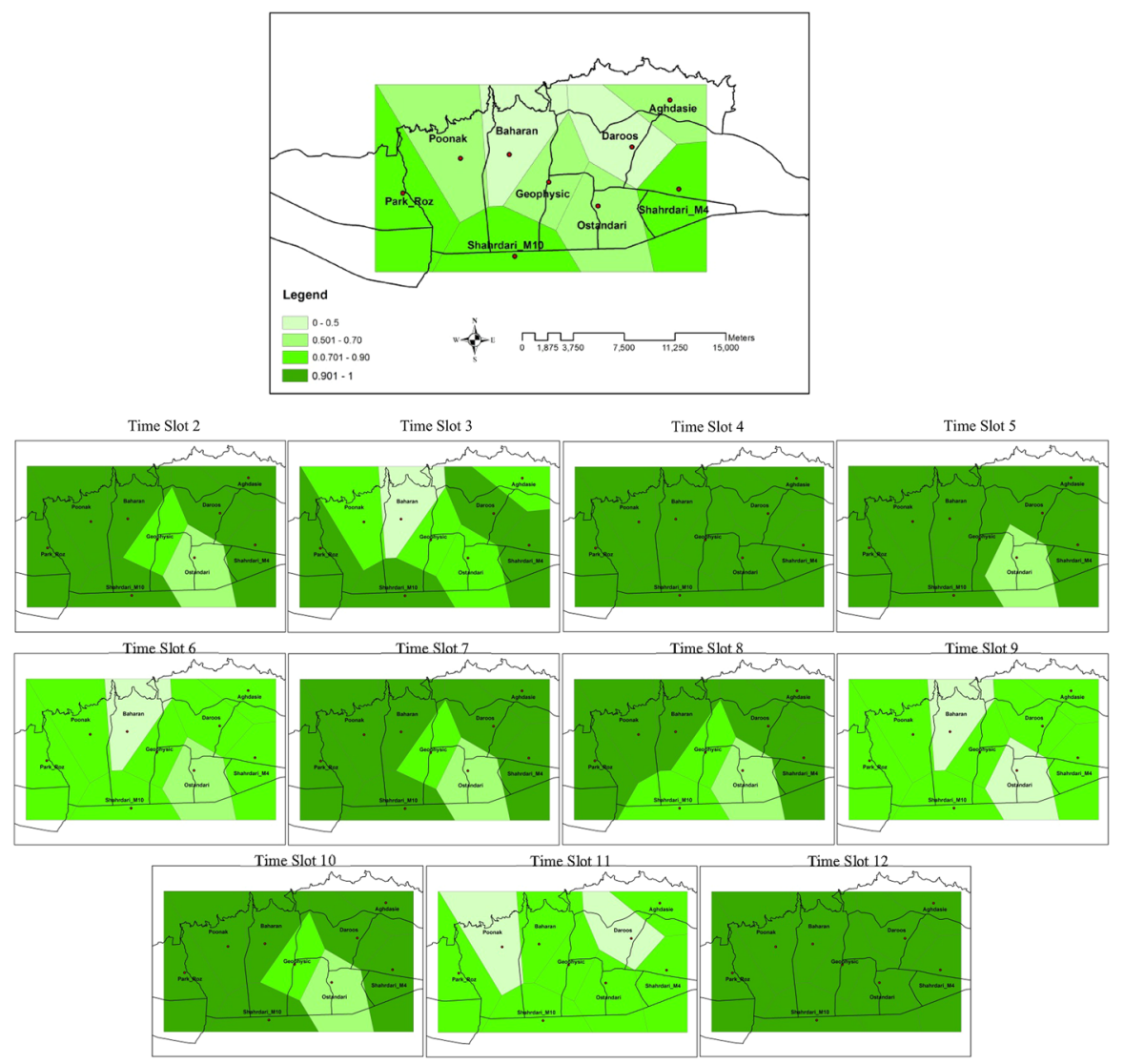

Fig. 9 Map of the VIF index for different time slots

where, in Eq. 7, "m" is the number of prevalent co-location patterns in each region and the weight function is the prevalence threshold (PI). The results of these three criteria for the case study are presented in Figs. 7, 8 and 9.

\section{Discussion}

Using Figs. 4 and 5, it can be seen that the most prominent and prevalent patterns in the case study are (ap2; tr1), (ap2; tr3), (ap2; tr2), (ap3; tp1), (ap3; wn1) and (ap3; tp1; wn1). These patterns are classified as sustained or time prevalent patterns, based on the predefined TPI criteria and classification conditions. The extracted patterns contain nearly all studied parameters, but it is worth discussing the results in more detail. First, for the air pollution parameter, there are only occurrences of air pollution classes 2 and 3. Since (Azizi 2011; Kavousi et al. 2013; Saadatabadi et al. 2012) had mentioned high air pollution as a major problem for Tehran, it had to be expected that most patterns are co-occurrences having higher levels of air pollution 
classes 2 and 3. It is also evident that with respect to air pollution as the pattern core element, traffic plays an important role. Since CO was used as air pollutant in this study and also due to the fact that the most important sources of this pollutant are motor cars, it makes CO a traffic-related pollutant (Azizi 2011; Safavi and Alijani 2006); afterward, it was also expected that there are prevalent patterns between air pollution and traffic. The other parameters participating in the above patterns are topography class $1(<1300 \mathrm{~m})$ and wind speed class $1(<6 \mathrm{knot})$. As (Safavi and Alijani 2006) explains, the Alborz Mountains located to the north and northeast of Tehran hamper westerly winds and cause air pollutants to remain in the city. Tehran on the southern slope of the Alborz chain lies in a semi-closed area (Fig. 3). Generally, the topography of Tehran slopes from north to south, although some height variations can be found in the city as well. This topographic configuration causes poor wind circulation with low wind speeds, trapping all pollutions in the city. Authors in (Saadatabadi et al. 2012) conclude that low wind speed is one of the most important meteorological factors causing high levels of $\mathrm{CO}$ concentration. Very clearly, the extracted patterns of the case study confirm the relationship between high air pollution and low wind speed. Additionally, temperature inversion occurrences further trap pollutants in the city, especially in central and southern parts of Tehran (Rahimi Ghoroghi 2012; Safavi and Alijani 2006), where the topography class is 1 (i.e., low topography). Therefore, the extracted pattern of air pollution class 3 (high) and topography class 1 (low) is a valid pattern.

Considering at Figs. 4 and 5, two observations can be made regarding the spatial and the temporal distribution of patterns, respectively. First, most of the patterns concentrate in the central parts of Tehran, that is, regions 2, 3, 6 and 7 shown in Fig. 3. This can be confirmed by the existing rules in Tehran to confront the air pollution problems, in this case, the Area Traffic Plan that exists for the central parts of Tehran. In addition, (Shad et al. 2009) confirms that air pollutants concentrate in the central and eastern parts of Tehran. Second, regarding the temporal distribution, it can be seen that in the first time slots of the series, there are relatively few patterns. Later on, the number of patterns increases until the tenth time slot and then decreases again.

Generally speaking, it shows that most prevalent patterns occur from the beginning of fall until the beginning of winter. On the other hand, except during the second time slot, low numbers of prevalent patterns occur in the spring, the beginning of summer and the end of winter. However, perhaps the kinds of patterns that exist in each time slot are more important than the number of patterns. In Fig. 4, it can easily be understood that most of the patterns in the period of the beginning of fall to the beginning of winter are those that co-occur with air pollutions of class 3 . As mentioned above, low wind circulation in Tehran is further aggravated by a meteorological situation named inversion, which occurs most frequently around this time of the year and causes more air pollution (Rahimi Ghoroghi 2012; Safavi and Alijani 2006). Conversely, in the spring, summer and the end of winter inversions are less frequent, and thus, patterns with air pollution class 3 are rare.

In addition, paying more attention to the extracted patterns shown in Fig. 5, it can be identified that air pollution class 3 ( $>5 \mathrm{ppm} \mathrm{CO}$ ) has a tendency to occur in the eastern parts of Tehran. As reported in (Rahimi Ghoroghi 2012), the prevalent wind 
direction in Tehran is westerly winds that move air pollution from the western parts of Tehran to the eastern parts; however, due to the presence of mountains, they remain blocked in the eastern parts, increasing the air pollution in that area, as found by (Shad et al. 2009). Repeatedly, this confirms the validity of this approach.

Considering the extracted patterns from the spatial point of view, it is clear that in time slots having a lower number of patterns, those patterns are more spatially concentrated compared to in time slots with more patterns. It seems plausible that whenever there are more patterns, the probability of those patterns covering a wider area is higher. While the extracted patterns follow this general rule in the spatial distribution, thus confirming the above findings, some additional information can also be learned. Specifically, as can be seen in Fig. 5, in time slots where only patterns between air pollution and traffic exist, the line lengths are smaller than in time slots with more parameters participating in patterns. This confirms the fact that the most important source of air pollution is traffic (Azizi 2011; Safavi and Alijani 2006), where the geometric centers of air pollution and traffic data lie close to each other. On the other hand, although low wind speed and topography have a close correlation with air pollution, because of the fact that those parameters are not the source of air pollution, geometric centers of air pollution and wind speed and topography, respectively, are not expected to lie as close as is the case for traffic.

In Fig. 8, feature density in different regions is showed. As can be seen, central parts of the city show higher concentrations of features due to the denser traffic network in these parts. Figure 9 depicts the density of prevalent co-occurrence patterns in each of the study regions. It can be seen that the central parts of the city, especially the "Ostandari," "Shahrdari_10" and "Geophysic" regions, respectively, have higher values in most of the VGF maps which shows that most of the occurring patterns are concentrated in these parts, which is in correspondence with our previous discussions. Considering Fig. 8 and 9, it is evident that the VIF index does not exhibit the same behavior as the VGF index. Using Fig. 9, it can be found that in most times, regions that have higher VGF values-that means more patterns occur in these regions-have lower VIF values, which seems plausible because whenever there are more patterns indices, the probability of the VIF index to be more balanced is higher.

One of the findings in this research is that although there is a relatively clear trend in pattern change over time, there are also exceptions from this trend. For example, the patterns of time slot 2 are against the existing trend. This may be due to some other temporal parameters affecting air pollution that have not been considered. It may also be caused by the fact that only 1 day per month was selected, which may cause outliers.

To find an optimal neighborhood radius, as well as the optimal spatial and temporal prevalence thresholds, is a challenge that lies outside the scope of this research, but it will be important in order to present the best results for a decision maker. Clearly, however, these quantities largely depend on the spatio-temporal process under study, and the parameters that govern this process. In this case, the process studied was air pollution, and the influencing factors were traffic volume, wind speed and topography. As explained in Sect. 5, given some knowledge of the 
process studied, it is then possible to select input parameter values (neighborhood radius, prevalence thresholds) that are adjusted to the process.

To conclude this discussion, an important contribution of the pattern extraction and visualization model is that decision makers can spatially and temporally track air pollution patterns in conjunction with the parameters affecting this process, see the types of relations occurring, their importance, their spatial and temporal distribution, change over time, and other related factors. Based on the results of this model, urban managers may change management procedures; for example, after analysis, they may change the Tehran Traffic Area temporally for each month. This would not only allow defining a realistic area for traffic control but would also reduce the difficulties of people in limited transportation areas.

\section{Conclusion and future work}

Based on the existing techniques for spatial co-location mining, a new method called RST-CoM for spatio-temporal co-occurrence pattern mining is developed. The proposed method has several important extensions. First, the pattern search is localized by indexing the data to a Voronoi tessellation prior to the co-location mining process, thus reducing the computational cost. Second, another property of the proposed method is that it considers all the feature types (point, line and polygon) in co-occurrence pattern mining, which undoubtedly is indispensable for real applications. Third, the method is developed to explore the temporal evolution of patterns. In contrast to other studies that consider features as moving objects, this research moved to a higher level and tracked the evolution of co-occurrence patterns in both space and time, considering the patterns as "moving objects." As a result, it is possible to classify the trends of co-occurrence patterns into six categories: sustained, emerging, dispersing, time prevalent, time variant and no pattern. To evaluate the proposed method, it is implemented with the $\mathrm{C \#}$ programming language and applied to a real case study: the extraction of air pollution patterns in relation to other influencing parameters, for the city of Tehran. The results of the experiments suggest that the proposed method may be used for real applications such as air pollution modeling. Finally, this study introduces new concepts to the domain of spatio-temporal co-occurrence pattern mining. To find co-occurrence patterns having the same emphasis on the spatial and temporal dimensions, respectively, is the other goal of this research that has been achieved.

In future studies, we would like to apply or extend our method of spatio-temporal co-occurrence pattern mining to different application domains such as noise pollution, crime, floods or car accidents. We also intend to extend our model for line and polygon as co-occurrence pattern core elements. Furthermore, future research may intend to develop and evaluate more comprehensible approaches of visualizing the extracted co-occurrence patterns in the spatio-temporal domain. In addition, we will try to combine co-occurrence pattern mining and LUR for a more complete model for air pollution modeling. 
Acknowledgments We are grateful of the Iran Meteorological Organization, the Tehran Air Quality Control Center, theTehran Traffic Control Center and the National Cartographic Center of Iran for providing our casestudy data.

\section{References}

Agarwal R, Srikant R (1994) Fast algorithms for mining association rules. In: Proceeding of the 20th International Conference on Very Large Data Bases (VLDB). pp 487-499

Akbari M, Samadzadegan F (2014) New regional co-location pattern mining method using fuzzy definition of neighborhood. Adv in Comput Sci: An Int J (ACSIJ) 3(3):32-37

Azizi MH (2011) Impact of traffic-related air pollution on public health: a real challenge. Arch Iran Med 14(2):139-143

Beelen R, Hoek G, Vienneau D, Eeftens M, Dimakopoulou K, Pedeli X, de Hoogh K et al (2013) Development of NO 2 and NO $\mathrm{x}$ land use regression models for estimating air pollution exposure in 36 study areas in Europe-the ESCAPE project. Atmos Env 72:10-23. doi:10.1016/j.atmosenv.2013. 02.037

Celik M (2011) Discovering partial spatio-temporal co-occurrence patterns. In: Proceeding of the 1st international conference on spatial data mining and geographical knowledge services, Fuzhou, China, 116-120. doi:10.1109/ICSDM.2011.5969016

Celik M, Kang JM, Shekhar S (2007) Zonal co-location pattern discovery with dynamic parameters. In: Proceeding of the seventh IEEE international conference on data mining, Omaha, NE, 433-438. doi:10.1109/ICDM.2007.102

Celik M, Shekhar S, Rogers JP, Shine JA (2008) Mixed-drove spatiotemporal co-occurrence pattern mining. IEEE Trans Knowl Data Eng 20(10):1322-1335. doi:10.1109/TKDE.2008.97

Celik M, Azginoglu N, Terzi R (2012) Mining periodic spatio-temporal co-occurrence patterns: a summary of results. In: Proceeding of the international symposium on innovations in intelligent systems and applications (INISTA), Trabzon, Turkey, 411-415. doi:10.1109/INISTA.2012.6247044

Champendal A, Kanevski M, Huguenot PE (2014) Air pollution mapping using nonlinear land use regression models. In: Murgante B et al (eds) Computational science and its applications-ICCSA 2014. Part III, LNCS 8581, Springer, Switzerland, pp 682-690. doi:10.1007/978-3-319-09150-1_50

Desmier E, Flouvat F, Gay D, Selmaoui-Folcher N (2011) A clustering-based visualization of colocation patterns. In: Proceedings of the 15th Symposium on international database engineering \& applications. 70-78. ACM. doi:10.1145/2076623.2076633

Ding W, Jiamthapthaksinl R, Parmar R, Jiang D, Stepinski TF, Eick CF (2008) Towards region discovery in spatial datasets. In: Proceeding of the Pacific-Asia conference on knowledge discovery and data mining (PAKDD). Osaka, Japan 88-99. doi:10.1007/978-3-540-68125-0_10

Goodchild MF (2003) The fundamental laws of GIScience. invited talk at university consortium for geographic information science. University of California, Santa Barbara

Gudmundsson J, Kreveld MV (2006) Computing longest duration flocks in trajectory data. In: Proceeding of the ACM international symposium on geographic information systems. Virginia, USA. 35-42. doi:10.1145/1183471.1183479

Huang Y, Shekhar S, Xiong H (2004) Discovering co-location patterns from spatial datasets: a general approach. IEEE Trans Knowl Data Eng 16(12):1472-1485

Huang Y, Zhang L, Zhang P (2008) A framework for mining sequential patterns from spatio-temporal event datasets. IEEE Trans Knowl Data Eng 20(4):433-448. doi:10.1109/TKDE.2007.190712

Icking C, Klein R, Kollner P, Ma L (2003) Java applets for the dynamic visualization of voronoi diagrams. comput. sci. in perspect. Lect Notes Comput Sci 2598:191-205. doi:10.1007/3-54036477-3_14

Kanaroglou PS, Adams MD, De Luca PF, Corr D, Sohel N (2013) Estimation of sulfur dioxide air pollution concentrations with a spatial autoregressive model. Atmos Env 79:421-427. doi:10.1016/j. atmosenv.2013.07.014

Kavousi A, Sefidkar R, Alavimajd H, Rashidi Y, Khonbi ZA (2013) Spatial analysis of CO and PM10 pollutants in Tehran city. J Paramed Sci (JPS) 4(3):41-50 (ISSN: 2008-4978)

Manikandan G, Srinivasan S (2012a) Mining of spatial co-location pattern implementation by FP growth. Indian J Comput Sci Eng (IJCSE) 3(2):344-348 (ISSN: 0976-5166) 
Manikandan G, Srinivasan S (2012b) Mining spatially co-located objects from vehicle moving data. Eur J Sci Res 68(3):352-366 (ISSN: 1450-216X)

Miller HJ, Han J (2009) Geographic data mining and knowledge discovery, 2nd edn. CRC Press, published, London, p 486

Mohan P, Shekhar S, Shine JA, Rogers JP (2010) Cascading spatiotemporal pattern discovery: a summary of results. In: Proceeding of the SIAM international conference on data mining (SDM): pp 327-338

Priya G, Jaisankar N, Venkatesan M (2011) Mining co-location patterns from spatial data using rulebased approach. Int J Glob Res Comput Sci 2(7):58-61

Qian F, He Q, He J (2009a) Mining spread patterns of spatio-temporal co-occurrences over zones. In: Proceedings of the international conference on computational science and applications. 686-701. doi:10.1007/978-3-642-02457-3_57

Qian F, Yin L, He Q, He J (2009b) Mining spatio-temporal co-location patterns with weighted sliding window. In: Proceedings of the IEEE international conference on intelligent computing and intelligent systems ICIS 2009.181-185. doi:10.1109/ICICISYS.2009.5358192

Rahimi Ghoroghi N (2012) Evaluation of geographical factors on Tehran air pollution and its relation with temperature inversion. In: Proceedings of the first conference of air and noise pollution management. Tehran, Iran. http://www.civilica.com/Paper-CANPM01-CANPM01_039.html. (In Persian)

Saadatabadi AR, Mohammadian L, Vazifeh A (2012) Controls on air pollution over a semi-enclosed basin, Tehran: a synoptic climatological approach Iran. J Sci \& Technol (IJST) 4:501-510

Safavi SY, Alijani B (2006) Evaluation of geographical parameters in Tehran air pollution. Geogr Res J 58:99-112 (In Persian)

Shad R, Mesgari MS, Shad A (2009) Predicting air pollution using fuzzy genetic linear membership kriging in GIS. Comput, Environ Urban Syst 33(6):472-481

Shekhar S, Huang Y, Xiong H (2001) Discovering spatial co-location patterns: a summary of results. In: Proceeding of the 7th international symposium on spatial and temporal databases (SSTD). Redondo Beach, CA, USA. doi:10.1007/3-540-47724-1_13

Wan Y, Zhou J (2008) KNFCOM-T: a k-nearest features-based co-location pattern mining algorithm for large spatial data sets by using T-trees. Int J Bus Intell Data Min 3(4):375-389. doi:10.150/IJBIDM. 2008.022735

Xiao X, Xie X, Luo Q, Ma W (2008) Density based co-location pattern discovery. In: Proceeding of the ACM SIGSPATIAL international conference on advances in geographic information systems (ACM-GIS). Irvine, CA, USA. doi:10.1145/1463434.1463471

Xiong H, Shekhar S, Huang Y, Kumar V, Ma X, Yoo JS (2004) A framework for discovering co-location patterns in data sets with extended spatial objects. In: Proceeding of the 2004 SIAM international conference on data mining (SDM'04). Lake Buena Vista, FL.78-89

Yoo JS, Bow M (2011) Mining top-k closed co-location patterns. In: Proceeding of the IEEE international conference on spatial data mining and geographical knowledge services (ICSDM). Fuzhou 100-105. doi:10.1109/ICSDM.2011.5969013

Yoo JS, Shekhar S (2005) A partial join approach for mining co-location patterns. In: Proceeding of the ACM SIGSPATIAL international conference on advances in geographic information systems (ACM-GIS). 241-249. doi:10.1145/1032222.1032258

Yoo JS, Shekhar S (2006) A join-less approach for mining spatial co-location patterns. IEEE Trans Knowl Data Eng 18(10):1323-1337. doi:10.1109/ICDM.2005.8 